\title{
TeV Cosmic-Ray Anisotropy from the Magnetic Field at the Heliospheric Boundary
}

\author{
V. López-Barquero ${ }^{1}$, S. Xu ${ }^{2}$, P. Desiati ${ }^{3,4}$, A. Lazarian ${ }^{4}$, N. V. Pogorelov ${ }^{5,6}$, and H. Yan ${ }^{7,8}$ \\ ${ }^{1}$ Department of Physics, University of Wisconsin, Madison, Wisconsin 53706, USA \\ 2 Department of Astronomy, School of Physics, Peking University, Beijing 100871, China \\ ${ }^{3}$ Wisconsin IceCube Particle Astrophysics Center (WIPAC), University of Wisconsin, Madison, WI 53703, USA \\ ${ }^{4}$ Department of Astronomy, University of Wisconsin, Madison, WI 53706, USA \\ ${ }^{5}$ Department of Physics, University of Alabama in Huntsville, Huntsville, AL 35899, USA \\ ${ }^{6}$ Center for Space Plasma and Aeronomic Research, University of Alabama in Huntsville, 320 Sparkman Dr., Huntsville, AL 35805, USA \\ ${ }^{7}$ DESY, Platanenallee 6, D-15738 Zeuthen, Germany \\ ${ }^{8}$ Institut fur Physik und Astronomie, Universität Potsdam, D-14476 Potsdam-Golm, Germany \\ Received 2017 February 16; revised 2017 May 1; accepted 2017 May 4; published 2017 June 14
}

\begin{abstract}
We performed numerical calculations to test the suggestion by Desiati and Lazarian that the anisotropies of TeV cosmic rays may arise from their interactions with the heliosphere. For this purpose, we used a magnetic field model of the heliosphere and performed direct numerical calculations of particle trajectories. Unlike earlier papers testing the idea, we did not employ time-reversible techniques that are based on Liouville's theorem. We showed numerically that for scattering by the heliosphere, the conditions of Liouville's theorem are not satisfied, and the adiabatic approximation and time-reversibility of the particle trajectories are not valid. Our results indicate sensitivity to the magnetic structure of the heliospheric magnetic field, and we expect that this will be useful for probing this structure in future research.
\end{abstract}

Key words: cosmic rays - magnetic fields - magnetohydrodynamics (MHD) - solar wind - Sun: heliosphere

\section{Introduction}

Cosmic rays (henceforth CRs) with energy below $10^{18} \mathrm{eV}$ have a gyroradius smaller than the galactic disk thickness of about $300 \mathrm{pc}$, with energy-dependent confinement within the Milky Way. The topics related to the origin, propagation, and acceleration of CRs are still debated in spite of the long history of relevant studies (see the excellent textbook by Longair 2011 and references therein). However, it is generally accepted that most of the galactic CRs are being accelerated by supernova shocks. Some percentage of the CRs can be accelerated by magnetic reconnection (de Gouveia dal Pino \& Lazarian 2005). ${ }^{9}$ Spatially, supernovae are correlated to star-forming regions, so the distribution of CRs is affected by that of their sources, but it is modified by propagation through the galactic magnetic field. Frequently the magnetic field in the Galaxy is described as being composed of a global regular component (see, e.g., Jansson \& Farrar 2012a, 2012b), large-scale coherent (on the order of $10-100 \mathrm{pc}$ ) structures, and the ubiquitous turbulent component (with wide spatial-scale inertial range with amplitude following a Kolmogorov power spectrum). This is, however, an approximation, with the availability of the modern theory of magnetohydrodynamic (MHD) turbulence (see Brandenburg \& Lazarian 2013 for a review) predicting a more sophisticated picture, with compressible and incompressible modes having their own cascades (Goldreich \& Sridhar 1995), henceforth GS95 (Lithwick \& Goldreich 2001; Cho \& Lazarian 2002, 2003; Kowal et al. 2009). For sub-Alfvénic turbulence, which is typical within quiescent regions of the interstellar medium (ISM), the transition from weak turbulence to strong Alfvénic turbulence takes place (Lazarian \& Vishniac

\footnotetext{
9 Magnetic reconnection becomes fast (i.e., independent of resistivity) in turbulent media (Lazarian \& Vishniac 1999; Kowal et al. 2009, 2012; see also a review by Lazarian et al. 2015 and references therein). A similar acceleration mechanism that appeals to tearing is discussed in a later publication by Drake et al. (2006).
}

1999; Galtier et al. 2000). The latter has the Kolmogorov-type spectrum $\sim k^{-5 / 3}$. However, this spectrum is strongly anisotropic, and therefore the scattering by Alfvénic turbulence injected at large scale is marginal (Chandran 2000; Yan \& Lazarian 2002), with fast modes identified by Yan \& Lazarian (2002) as the major scattering component induced by the galactic turbulent cascade.

An additional scattering emerges from CR instabilities. Streaming instability has been long considered an important component of CR propagation physics (see Cesarsky 1980 for a review). Particle streaming was employed in models such as the leaky box model of propagation to explain the high isotropy of observed CRs. In that model, it was assumed that the streaming instability was suppressed in the partially ionized galactic disk and acts to scatter and return CRs as they enter the partially ionized galactic halo. The streaming of particles outside the disk was able to naturally explain the observed dipole anisotropies of the observed CR distribution. This model was later challenged in Farmer \& Goldreich (2004), who performed calculations of the streaming instability damping by the ISM turbulence and concluded that the streaming is not expected to take place for the levels of turbulent damping that they adopted. More recently, this conclusion was questioned in Lazarian (2016), where it was shown that for typical halo conditions, scattering instability takes place in the galactic halo. Additional instabilities of CRs (see Lazarian \& Beresnyak 2006; Yan \& Lazarian 2011) can act as additional sources of $\mathrm{CR}$ isotropization. In this paper, we assume that the influence of scattering induced by the TeV CR instabilities in the vicinity of the heliosphere is negligible.

The limitation of the traditional models of CR propagation is not only due to scattering physics. In fact, at scales less than the turbulent injection scale, the particles following magnetic field lines experience super-diffusion with respect to the direction of the mean field (Lazarian \& Yan 2014). Such effects can be strongly distorted if the synthetic data cubes are used. 
Therefore, in what follows, we use only the data cubes obtained by direct MHD numerical simulations.

This paper continues our numerical studies of the origin of CR anisotropies observed at Earth. The first paper, LópezBarquero et al. (2016; hereafter referred to as LX16), dealt with the effects of interstellar turbulence on the CR propagation, and it did not take into account the strong perturbations induced by the heliosphere. In this paper, on the contrary, we focus our attention on the effects arising from the CR interactions with the heliosphere. The idea that the heliosphere can produce strong scattering on CRs, which could be important for explaining the observed high-energy CR anisotropies, was first suggested in Desiati \& Lazarian (2013; hereafter referred to as DL13). This idea was later tested numerically in Schwadron et al. (2014) and Zhang et al. (2014). The difference between our paper and the earlier studies is that we do not assume that Liouville's theorem and the backtracking of particles are valid. In fact, in this paper, we show that the conditions of Liouville's theorem are not satisfied, due to the scattering at the heliospheric boundary. Therefore we adopt a much more time-consuming Monte-Carlo approach with the forward tracking of particles.

The paper is organized as follows. In Section 2, the problem of observed anisotropy of CRs is formulated along with ways to address it, while in Section 3.1 the long tail heliospheric model used in this study is described. Section 3 describes the particle integration method used and which CR particles are used in the study. In Section 4, we discuss the validity of applying Liouville's theorem in the context of this work. Results are presented in Section 5 and discussed in Section 6. Concluding remarks follow in Section 7.

\section{The Problem of Anisotropies and Corresponding Approaches}

Particle energy roughly determines which spatial scale is the most dominant in shaping the characteristics of their distribution. Galactic CRs in the energy range below about $50 \mathrm{GeV}$ are strongly affected by modulations of the inner heliospheric magnetic field, in correlation with solar cycles (see, e.g., Florinski et al. 2013, 2015; Potgieter 2013; Manuel et al. 2014). Above $50 \mathrm{GeV}$, the modulation in the CR energy spectrum is negligible; however, the effects of long-term solar cycles on particle distribution are still observed up to an energy of few hundred GeV (Munakata et al. 2010; Kozai et al. 2014). The gyroradius of 10- to $100-\mathrm{GeV}$ CR particles in the interplanetary magnetic field of $<1 \mu \mathrm{G}$ is typically smaller than the size of the termination shock (about 80-90 au; see Pogorelov et al. 2015). This makes it possible for those particles to be spatially redistributed according to the modulating solar wind-induced perturbations on the magnetic field.

At $\mathrm{TeV}$ energies, a scale transition occurs. The typical particle gyroradius is larger than the size of the termination shock; therefore the influence of inner heliospheric magnetic fields on the CR distribution is negligible. In fact, solar cycle modulations are subdominant in this energy range. However, $\mathrm{TeV}$ galactic CRs coming from the ISM, where the local interstellar magnetic field (LIMF) is $\sim 3 \mu \mathrm{G}$, and propagating into the heliosphere, have gyroradius on the order of $100 \mathrm{au}$, which is smaller than the estimated transverse size of the heliosphere of about 600 au and shorter than the estimated length of the heliospheric tail of a few thousand au (Pogorelov et al. 2006, 2015; Pogorelov 2016). From this scaling relationship, $\mathrm{TeV} \mathrm{CRs}$ are expected to be influenced by the heliospheric magnetic field (see Desiati \& Lazarian 2013, hereafter referred to as DL13). At an energy scale of tens of $\mathrm{TeV}$, the gyroradius of the lightest CR particles starts to exceed the heliosphere's transverse size, thus decreasing its influence; nonetheless, these particles will still experience the influence of the perturbation created by the heliosphere on the local interstellar medium (LISM) and the effects of the heliotail's length on their propagation. At higher energies, the arrival directions of the CRs are influenced by their propagation through the interstellar magnetic field (see LX16) and by the distribution of their sources in the Galaxy.

It is thus expected that CRs with energy below several tens of $\mathrm{TeV}$ are influenced by the heliosphere to some extent. The actual degree of such an effect depends on the properties of the heliosphere, such as its size and the magnetic structure, the presence of magnetic perturbations or instabilities at the boundary with the ISM (e.g., at the flanks of the heliosphere), the large-scale perturbation of the LISM due to the heliosphere, and the mass composition (or better the rigidity ${ }^{10}$ ) of the CR particles. If the heliosphere has the effect to redistribute the $\mathrm{TeV}$ CR-arrival direction distribution, compared with that shaped by interstellar propagation, all those details need to be properly understood and integrated into a comprehensive numerical particle trajectory integration.

From an observational point of view, a statistically significant anisotropy has been observed by a variety of experiments, sensitive to different energy ranges (from tens of $\mathrm{GeV}$ to a few $\mathrm{PeV}$ ), located on or below the Earth's surface in the Northern Hemisphere (Nagashima et al. 1998; Hall et al. 1999; Amenomori et al. 2005, 2006, 2011; Guillian et al. 2007; Abdo et al. 2009; Aglietta et al. 2009; Zhang et al. 2009; Munakata et al. 2010; Cui et al. 2011; de Jong et al. 2011; Bartoli et al. 2015) and in the Southern Hemisphere (Abbasi et al. 2010, 2011, 2012; Aartsen et al. 2013, 2016).

The global anisotropy appears to change with energy in a nontrivial way. From about $100 \mathrm{GeV}$ to tens of $\mathrm{TeV}$, it has an approximately consistent structure at the largest scale, although its measured amplitude increases with energy. Above a few tens of $\mathrm{TeV}$, the observed progressive change in the anisotropy topology may indicate a transition between two processes shaping the particles' arrival distribution at Earth-for instance, the transition from heliospheric-dominated to ISM-dominated influence, which culminates around $100 \mathrm{TeV}$ (as observed in Aartsen et al. 2016 and discussed in DL13).

However, the change in topology of the CR anisotropy as a function of energy can have different origins as well. In the scenario of particles in homogeneous and isotropic diffusion, the CR density gradient, and therefore the induced spatial anisotropy, has a dipolar shape. The direction of the dipole is expected to point toward the strongest source of the observed CRs, and its amplitude is expected to depend on the diffusion coefficient. At different energies, the strongest contribution to the observations can shift from one source to another, thus changing the orientation of the dipole (Erlykin \& Wolfendale 2006; Blasi \& Amato 2012; Ptuskin 2012; Pohl \& Eichler 2013; Sveshnikova et al. 2013; Savchenko et al. 2015;

\footnotetext{
${ }^{10}$ The rigidity of a charged particle is a measure of its momentum, and it refers to the fact that a higher momentum particle has a higher resistance to deflection by a magnetic field. It is defined as $R=r_{L} B c=E / Z e$, with $r_{L}$ as the particle gyroradius and $B$ as the magnetic field (see Wiedemann 2015). A $1 \mathrm{TeV}$ proton and a $26 \mathrm{TeV}$ iron nucleus have a rigidity of $1 \mathrm{TV}$.
} 
Ahlers 2016). The difficulty with this scenario is that particle diffusion in the ISM is expected to be anisotropic (i.e., fast along and slow across the magnetic field lines). A misalignment between the CR density gradient and the regular galactic magnetic field prevents pointing to any specific source, and it would suppress the anisotropy amplitude, depending on the misalignment angle (Effenberger et al. 2012; Kumar \& Eichler 2014; Schwadron et al. 2014; Mertsch \& Funk 2015). Since the ratio of perpendicular to parallel diffusion is likely to depend on energy (depending on the magnetic field geometric configuration), the change in orientation of the anisotropy is also linked to the properties of the interstellar magnetic field itself.

The observed anisotropy cannot be described with a simple dipole component. The actual distribution is a combination of several angular scales (Amenomori et al. 2007; Abdo et al. 2008; Abbasi et al. 2011; Bartoli et al. 2013; Abeysekara et al. 2014; Aartsen et al. 2016) that can be studied by decomposing it into individual spherical harmonic contributions. This makes it possible to determine the angular power spectrum of the observed arrival distribution. As reported by experimental observations, most of the power is concentrated in the large-scale anisotropy structure, which includes dipole, quadrupole, and octupole. Such contributions are likely affected by the limited field of view of the experiments and also by biases that limit the observation at large scale (see, e.g., Ahlers et al. 2016). About 1\% of the power is distributed across small-scale structures in the arrival direction distribution (where there is no bias due to the field of view). Small angular scale anisotropy features correspond to regions where CR flux has large gradients in a relatively localized area in the field of view of the observations (on the order of $10^{\circ}$ ). Such regions can be stochastically produced by scattering processes of CRs in the ISM magnetic turbulence within the particle mean free path, as discussed in Giacinti \& Sigl (2012), Ahlers (2014), Ahlers \& Mertsch (2015), and our companion paper LX16. Such scattering processes have the effect of decomposing a large-scale particle density gradient into small-scale components. This process constitutes an important contribution to the power spectrum, and it is certainly compatible with observations. However, it is possible to argue that some observed localized regions of $\mathrm{TeV} \mathrm{CR}$ excess appear to be correlated with features associated with the heliosphere. For instance, one of the localized excess regions observed in the northern equatorial sky appears to be correlated with the direction of the heliospheric tail (see, e.g., Amenomori et al. 2007; Abdo et al. 2008; Bartoli et al. 2013; Abeysekara et al. 2014). CRs observed within this localized region have an energy spectrum that is harder than that in the surrounding areas. It was proposed that reacceleration of CRs by magnetic reconnections in the heliospheric tail may be a possible explanation (Lazarian \& Desiati 2010; Desiati \& Lazarian 2012). Other localized regions are spatially correlated with the large angular gradient edge of relative intensity across the whole sky, with a possible link to heliospheric origin (as discussed in DL13).

Instabilities that develop dynamically at the boundary between the heliosphere and the ISM (see Section 3.1), such as Rayleigh-Taylor and Kelvin-Helmholtz instabilities, can have an effect on the cosmic-ray arrival at Earth (Liewer et al. 1996; Zank et al. 1996, 2009; Zank 1999; Florinski et al. 2005; Pogorelov et al. 2006; Borovikov et al. 2008; Shaikh \& Zank 2010; Pogorelov et al. 2015). Specifically, if they have spatial scales on the order of 10-100 au, they can induce scattering processes on multi-TeV-scale CRs that cross the heliosphere. Therefore, if an instability affects the configuration of the heliopause, this can potentially have an impact on the CR distribution. However, currently the information on the exact characteristics of the heliopause is limited; thus more direct measurements are needed to assess the definite nature of these instabilities. The possibility that strong resonant scattering processes cause a redistribution of the CR-arrival direction distribution was already discussed in DL13. As mentioned, other authors have studied the effects of the heliosphere (Schwadron et al. 2014; Zhang et al. 2014) or, in general, of astrospheres (Scherer et al. 2016) on the distribution of $\mathrm{TeV}$ CRs. In addition, magnetic field reconnection plays an important role in the heliopause. For instance, turbulent magnetic reconnection (Lazarian \& Vishniac 1999, see also Lazarian et al. 2015 and ref. therein) can induce the first order acceleration of energetic particles (de Gouveia dal Pino \& Lazarian 2005; Lazarian 2005; Drake et al. 2006), which was shown to be relevant to the heliospheric settings (Lazarian \& Opher 2009; Drake et al. 2010).

The heliospheric model used in the present work makes use of ideal MHD treatment of ions and of a kinetic multi-fluid description of neutral interstellar atoms penetrating into the heliosphere (Pogorelov et al. 2013). This model incorporates the heliospheric tail up to a distance of approximately $4000 \mathrm{au}$, which does not cover the maximum possible extension (see Section 3.1). In this study, the possibility that resonant scattering processes may have a strong effect on redistributing TeV CR-arrival direction distribution is critically discussed. The relevant points of the present study are to dispute whether Liouville's theorem can actually be used as a tool to determine the particle trajectories affected by the heliospheric magnetic field and whether the heliosphere itself imprints a strong effect on the cosmic particles crossing it. If magnetic fields change significantly within gyroradius spatial scale, the geometry of particle trajectories may be highly sensitive to the actual initial conditions (i.e., they may have a chaotic nature). In such a case, the application of Liouville's theorem is not warranted, and particle distribution may follow a different scaling. In general, application of Liouville's theorem must to be investigated case by case. In what follows, we use direct numerical simulations of CR propagation using the numerical results of heliospheric simulations and without any use of Liouville's theorem.

\section{Cosmic-Ray Propagation in the Heliosphere}

In this section, the description of the heliospheric magnetic field model used in the present study is laid out; then the strategy and method used to numerically integrate the particle trajectories through the heliosphere are described.

\subsection{Heliosphere Magnetic Field Model}

The heliosphere is formed when the solar wind (SW) collides with the local interstellar medium (LISM). In an ideal magnetohydrodynamic formulation of the problem, the SWLISM interaction necessarily creates a tangential discontinuity that separates the plasmas originating at these two sources. This discontinuity is called the heliopause (HP). The HP extends thousands of astronomical units (au) from the Sun. As with any tangential discontinuity, the HP is subject to hydrodynamic instabilities-for example, the Kelvin-Helmholtz

$(\mathrm{KH})$ 
instability (see, e.g., Ruderman \& Fahr 1995; Chalov 1996; Belov \& Ruderman 2010; Ruderman \& Belov 2010). Moreover, the HP nose is subject to Rayleigh-Taylor (RT) instability. The role of gravity in this case is played by the momentum-exchange terms in the MHD equations describing the plasma flow in the presence of charge exchange (Liewer et al. 1996; Zank 1999). Linear analysis (Avinash et al. 2014) of the RT instability performed in an idealized formulation showed that perturbations grow unconditionally while they are small. Nonlinear, numerical investigations of the RT instability have been performed by Florinski et al. (2005) and Borovikov et al. (2008) in an axially symmetric case, and by Borovikov \& Pogorelov (2014) in a realistically three-dimensional formulation. It was demonstrated in the latter paper that the heliospheric magnetic field (HMF) can damp the RT instability. However, the HMF becomes rather small occasionally in the course of solar cycle, so the instability results in a substantial mixing of LISM and SW plasma at the nose of the heliopause. Interestingly, Borovikov \& Pogorelov (2014) show that RT instability may reveal itself also at the HP flanks, but it is caused by charge exchange with secondary neutral atoms born inside the heliosphere. As a result, the HP surface bounding the heliotail is subject to the mixture of $\mathrm{KH}$ and $\mathrm{RT}$ instabilities.

Since the KH instability is of a convective type, with the perturbation amplitude growing as a function of distance along the HP, plasma mixing as well as diffusion processes would eventually destroy the HP. However, it appears that charge exchange is a dominant process that results in continuous elimination of the hotter SW ions, with cooler ions possessing properties of the LISM $\mathrm{H}$ atoms. As shown by Izmodenov and Alexashov (2003) in an axially symmetric case and Pogorelov et al. (2015) in 3D, this makes the SW flow superfast magnetosonic (its Mach number calculated using the fast magnetosonic speed is greater than 1) at about $4000 \mathrm{au}$. In the investigation presented in this paper, we use one of the heliotail models described in Pogorelov et al. (2015). This model is based on a self-consistent solution of the ideal MHD equations, with appropriate source terms due to charge exchange between ions and neutrals, to describe the flow of plasma and the Boltzmann equation to describe the transport of neutral atoms. To avoid issues related to the heliospheric current sheet, this model assumes a unipolar distribution of the HMF inside the heliosphere. It shows initial collimation of the SW plasma inside the Parker spiral field lines bent tailward by the flow, as predicted by Yu (1974). The spiral field being kink-instable (Roberts 1956; Pogorelov et al. 2015), the reason for such collimation disappears at about 800 au from the Sun. In contrast with Yu (1974) and multi-fluid simulations of Opher et al. (2015), no separation of the HP into two lobes occurs in Pogorelov et al. (2015). This is because multi-fluid models substantially depress charge exchange across the lobe separation region. Such entirely hydrodynamic artifacts are impossible if atoms are treated kinetically. We assume the following properties of the LISM: temperature $T_{\infty}=6300 \mathrm{~K}$, velocity $V_{\infty}=23.2 \mathrm{~km} \mathrm{~s}^{-1}$, proton density $n_{\infty}=0.082 \mathrm{~cm}^{-3}, \mathrm{H}$ atom density $n_{H \infty}=0.172 \mathrm{~cm}^{-3}$, and magnetic field strength $B_{\infty}=3 \mu \mathrm{G}$. The LISM flow comes from the direction $(\lambda, \beta)=\left(79^{\circ},-5^{\circ}\right)$, while the $\boldsymbol{B}_{\infty}$ vector arises from $(\lambda, \beta)=\left(225^{\circ}, 44^{\circ}\right)$ in the ecliptic coordinate system (Zank et al. 2013). The SW is assumed to be spherically symmetric with the following properties at $1 \mathrm{au}$ : plasma density $n_{\mathrm{SW}}=7.4 \mathrm{~cm}^{-3}$, temperature $T_{\mathrm{SW}}=51,100 \mathrm{~K}$, radial velocity
$V_{\mathrm{SW}}=450 \mathrm{~km} \mathrm{~s}$, and radial magnetic field component $B_{R}=37.5 \mu \mathrm{G}$. The HMF is assumed to be Parker's at $1 \mathrm{au}$.

\subsection{Particle Trajectory Integration}

Similar to the work in LX16, this study is performed by integrating particle trajectories in the magnetic field described in Section 3.1, using the set of six-dimensional ordinary differential equations

$$
\begin{gathered}
\frac{d \boldsymbol{p}}{d t}=q(\boldsymbol{u} \times \boldsymbol{B}) \\
\frac{d \boldsymbol{r}}{d t}=\boldsymbol{u},
\end{gathered}
$$

describing the Lorentz force with $\boldsymbol{u}$ the particle velocity, $\boldsymbol{r}$ its position vector, and $\boldsymbol{p}$ the momentum. For $\boldsymbol{B}$, we use one steady-state realization of the heliospheric magnetic field described in Section 3.1. As in LX16, the equations are integrated using the Bulirsch-Stoer integration method (Press et al. 1986) with adaptive time step. At each integration step, the magnetic field is interpolated using a $3 \mathrm{D}$ cubic spline, and integration is stopped when particles cross the border of the simulation box. The choice of one specific realization of the magnetic field is justified by the fact that particle velocity is much larger than the plasma Alfvén velocity; thus induced electric fields can be neglected.

Figure 1 shows the meridional projection of the heliospheric model snapshot (described in Section 3.1) used in this study. At the top, the figure shows high-resolution visual details of the magnetic fields (color code in units of $\mu \mathrm{G}$ ) with the axes in units of au. At the bottom, the figure shows the magnetic field lines (coarse color code in units of $\mu \mathrm{G}$ ). The initial simulation box is $320 \times 280 \times 280$ simulation grid points (the longer dimension to allow the inclusion of the heliospheric tail) where each grid point corresponds to $20 \mathrm{au}$. This is equivalent to 6400 $\mathrm{au} \times 5600 \mathrm{au} \times 5600 \mathrm{au}$. The model has a varying resolution, depending on the region within the heliosphere, with the highest resolution in the region around the Sun, where it is $1 \mathrm{au}$ (0.05 simulation grid points). The model includes magnetic instabilities, on spatial scales on the order of 10-100 au, that grow on the flanks at the boundary with the ISM, and a tail with length of approximately $4000 \mathrm{au}$. In the model, the uniform interstellar magnetic field outside of the heliospheric boundary has intensity of about $3 \mu \mathrm{G}$. For the study presented in this work, the original simulation box is extended in such a way that a sphere centered at Earth and with radius 6000 au (300 simulation grid points) is inside it. In the extension of the simulation box, a uniform magnetic field with intensity $3 \mu \mathrm{G}$ and with the same direction as in the simulation is assumed.

In order to calculate the distribution of CRs at Earth after their propagation across the interstellar and heliospheric magnetic field, there are two possible methods that can be followed. One is to integrate a large number of particle trajectories (typically isotropically distributed) initiated on Earth and back-propagate (or back-track) them to outer space. The other method is to integrate particle trajectories initiated at a large distance from Earth and forward-propagate (or forward-track them, meaning directly propagate them according to the arrow of time) toward Earth. The back-propagation technique is based on the validity of Liouville's theorem, which states that particle density in phase space is conserved along the particle trajectories if conditions such as no collisional 

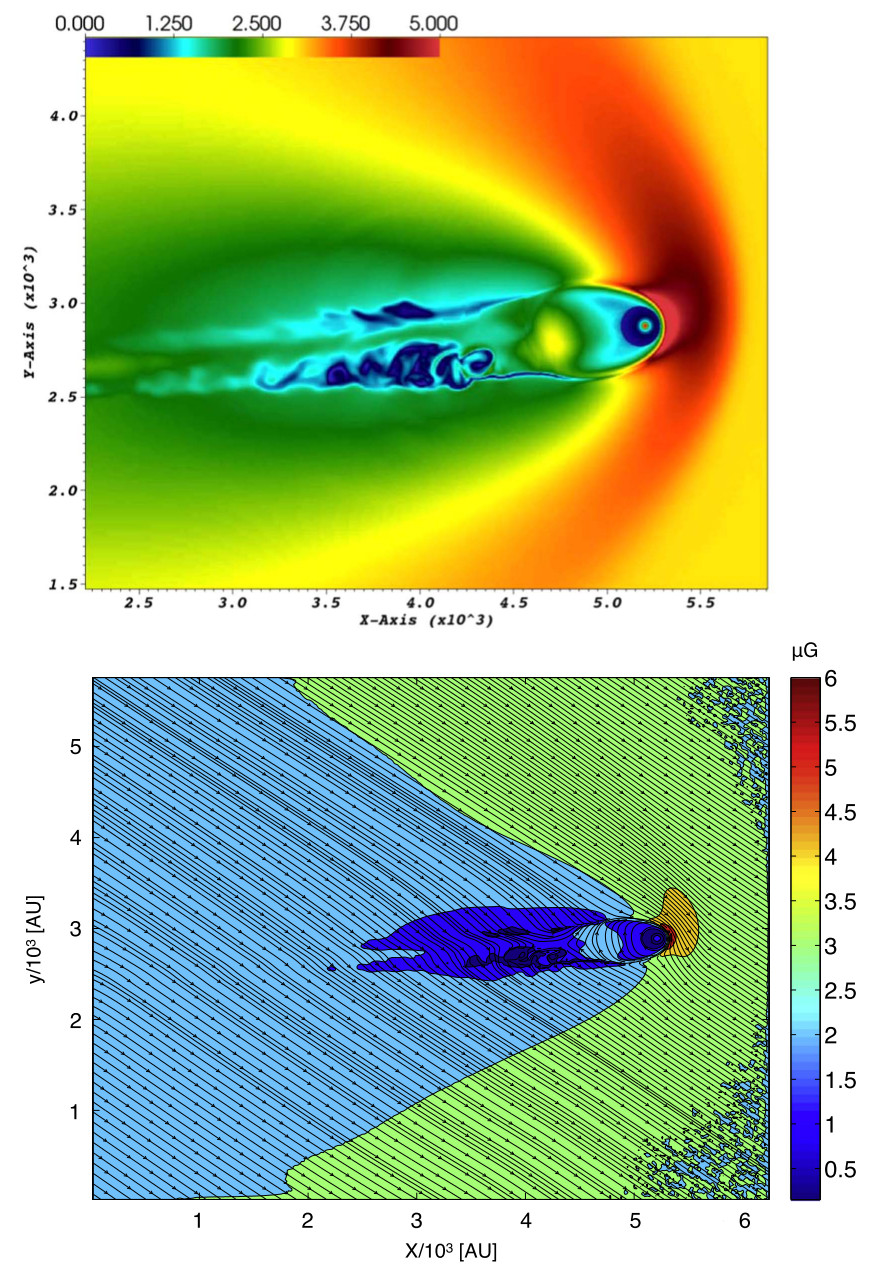

Figure 1. Meridional projection of the heliospheric magnetic field model described in Section 3.1. At the top, the figure shows high-resolution visual details of the magnetic fields (color code in units of $\mu \mathrm{G}$ ) with the axes in units of au. At the bottom, the figure shows the magnetic field lines (coarse color code in units of $\mu \mathrm{G}$ ) with the axes in units of au. The simulation box is $320 \times 280 \times 280$ grid points, corresponding to $6400 \mathrm{au} \times 5600 \mathrm{au} \times 5600 \mathrm{au}$ (20 au/grid point)

scattering or no resonant collisionless scattering are satisfied (see Section 4). If the theorem conditions are valid, then particle trajectories can be time reversed. This method is very efficient, because it entails the integration of particle trajectories from the target back into outer space. Nevertheless, there is no particle loss, and only portions of space that are directly magnetically connected to the target location have nonzero particle density population. Therefore it is necessary to impose an initial anisotropy as a directional dependent weight in order to calculate the particle distribution at the target position. Such a weight function breaks the isotropy initially constructed and provides the anisotropy distribution transmitted back to the target position from the magnetically connected remote regions of space. By construction, this technique does not take into account the generation of anisotropy from particle escape during their propagation.

In the presence of turbulence or instabilities, magnetic fields can vary in spatial scales that are shorter than the particle gyroradius. This breaks adiabaticity and effectively induces collisional processes that may invalidate the application of Liouville's theorem. The validity of Liouville's theorem is extensively discussed in LX16. In Section 4 of this paper, it is argued that the theorem does not have the grounds to be applicable for this particular study of heliospheric effects, and therefore it cannot be utilized. In this case, therefore, the forward-propagation method is used. Such a technique is implicitly inefficient, because only a small fraction of the injected particles will make it to or near the target. As discussed in Pohl \& Rettig (2015) as well, this method naturally accounts for the role that $\mathrm{CR}$ escape has in shaping the anisotropy. There is no need to assume a global anisotropy at large distance (injected, for instance, by CRs diffusively propagated away from a source) to obtain anisotropy at Earth, since particles naturally stream along interstellar magnetic field lines and undergo scattering processes in magnetic turbulence or instabilities.

In this study, it is argued that scattering at the boundary between the heliosphere and the ISM breaks the particle trajectory reversibility in that those particles that escape without reaching Earth cannot be represented in a backpropagation calculation method. Yet those trajectory configurations occur and contribute to the overall shape of CR-arrival direction distribution. In addition, the distribution of CRs at Earth is reshaped by the heliospheric instabilities in a stochastic manner, and the exact individual features produced by this phenomenon may not be predicted; therefore a statistical approach is used (e.g., by calculating the angular power spectrum of the arrival distribution).

The inefficiency intrinsic to the forward-propagating methods is compensated by starting particle trajectory integration only from those areas in space where they have a significantly higher chance to reach the neighborhood of Earth, and by assuming a "larger size" of Earth to record the trajectories that arrive at the final target. Although this last assumption may lead to approximate results, it is sufficient to unveil the role that the heliosphere has on the propagation of $\mathrm{TeV}$ CRs, independent of the propagation history in the ISM. The actual prediction of the anisotropy details most probably results from fine-tuning of several effects, and it is not addressed in this work.

\subsection{Cosmic-Ray Composition}

An important aspect of this study is taking into account that CRs are not dominated by protons only, which is particularly true at energies in excess of $1 \mathrm{TeV}$. As shown in Gaisser et al. (2013) and references therein, the abundance of helium nuclei is already comparable to that of protons at the $\mathrm{TeV}$ energy range, and it starts to dominate at $10 \mathrm{TeV}$. Heavier particles become increasingly more important at higher energies as well. Figure 2 shows that the maximum CR particle gyroradius $r_{L}$, averaged over the CR composition, is smaller than that of only protons, the higher the contribution from heavier nuclei. Therefore the relevant quantity is not the CR particle energy but their rigidity, $R=r_{L} B c$. Particles with same rigidity have the same gyroradius $r_{L}$ in a given magnetic field $B$. Or, equivalently, an iron nucleus of energy $E$ has a gyroradius that is 26 times smaller than that of a proton with the same energy and in the same magnetic field B. In the energy range of $1-10 \mathrm{TeV}$, galactic CRs are approximately composed of a mix of protons, helium, and heavier atomic nuclei (Gaisser et al. 2013). CR composition is an important ingredient in the understanding of anisotropy, especially since heavier particles (i.e., with small rigidity) may have a non-negligible contribution, even at relatively high particle energy. 


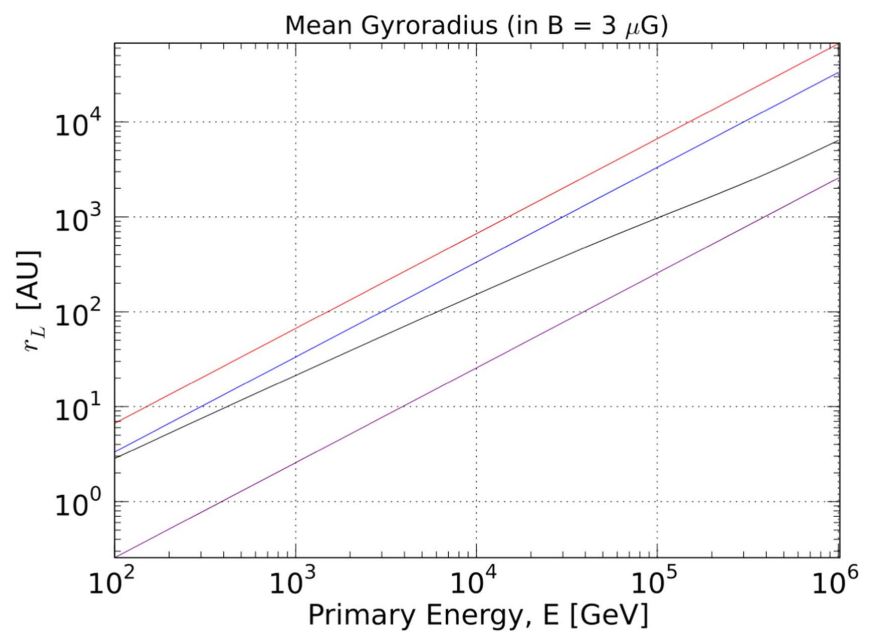

Figure 2. Cosmic-ray maximum gyroradius (or Larmor radius) $r_{L}$ in a $3 \mu \mathrm{G}$ magnetic field as a function of particle energy averaged over the observed mass composition (from Gaisser et al. 2013; black line). This is compared with that of protons (red line), of helium (in blue), and of iron nuclei (in purple). Note that due to the mass composition of cosmic rays, the average gyroradius is smaller than that for pure protons. This difference becomes important for energies in excess of about $1 \mathrm{TeV}$.

\subsection{Particle Data Sets}

In this study, trajectories for three types of particles are integrated, as shown in Table 1: protons, helium, and iron nuclei. Since the energy range of interest is approximately $1-10 \mathrm{TeV}$, two rigidity ranges are used here: $1 \mathrm{TV}$ (for all three particle types) and $10 \mathrm{TV}$ (for protons and helium nuclei). For each set, $1 \times 10^{6}$ particle trajectories are integrated with initial position on a sphere (labeled as injection sphere) of radius 6000 au (300 simulation grid points) centered on Earth, and with uniform direction distribution toward the inner volume of the sphere. The initial positions correspond to regions where particles streaming along the LIMF have a higher chance to reach the Earth's neighborhood. To account for scattering processes, the injection regions, on the interstellar-wind upstream and downstream directions of the LIMF, were initially identified by back-propagating particle trajectories from Earth across the heliospheric magnetic field (as shown in Figure 3). Once these main zones were identified, their extension was then expanded to account for the chance that particles initiated further away may reach Earth and yet maintain a manageable efficiency level. The regions where forward-propagated particle trajectories start are identified with a $30^{\circ} \times 30^{\circ}$ zone on the interstellar-wind upstream side of the heliosphere (i.e., on the lower right side of Figure 3 ) and with a $60^{\circ} \times 60^{\circ}$ zone on the interstellar-wind downstream side of the heliosphere (i.e., on the upper left side of Figure 3 ). For each set in Table 1 , out of $1 \times 10^{6}$ particles initiated in both regions, approximately $1 \times 10^{4}$ reach the vicinity of Earth (i.e., across a sphere centered on Earth, labeled as target sphere, of radius $200 \mathrm{au} ; 10$ simulation grid points).

The integrated trajectories from the sets of Table 1 are cumulated in the two rigidity range groups according to the relative mass composition from Gaisser et al. (2013): the $1 \mathrm{TV}$ and the $10 \mathrm{TV}$ rigidity scales. Table 1 shows the mean instantaneous gyroradius of the numerically integrated trajectories in the heliospheric magnetic field, and the corresponding rms (numerical values taken from the distributions in Figure 4).
Table 1

Physics Parameters of Simulation Sets

\begin{tabular}{llrrrr}
\hline \hline Set & Particle & $E_{p}$ & \multicolumn{1}{c}{$\left\langle r_{L}\right\rangle$} & $\begin{array}{c}\text { Injected } \\
\text { Particles }\end{array}$ & $\begin{array}{r}\text { Recorded } \\
\text { Particles }\end{array}$ \\
\hline 1 & Protons & $1 \mathrm{TeV}$ & $70.23 \pm 14.63 \mathrm{au}$ & $1 \times 10^{6}$ & 8758 \\
2 & Helium & $4 \mathrm{TeV}$ & $121.7 \pm 54.5 \mathrm{au}$ & $1 \times 10^{6}$ & 10416 \\
3 & Iron & $30 \mathrm{TeV}$ & $72.89 \pm 31.50 \mathrm{au}$ & $1 \times 10^{6}$ & 6065 \\
\hline 4 & Protons & $10 \mathrm{TeV}$ & $764.3 \pm 252.2 \mathrm{au}$ & $1 \times 10^{6}$ & 9789 \\
5 & Helium & $40 \mathrm{TeV}$ & $1235 . \pm 383 . \mathrm{au}$ & $1 \times 10^{6}$ & 8655 \\
\hline & & & $5 \times 10^{6}$ & 43683 \\
\hline
\end{tabular}

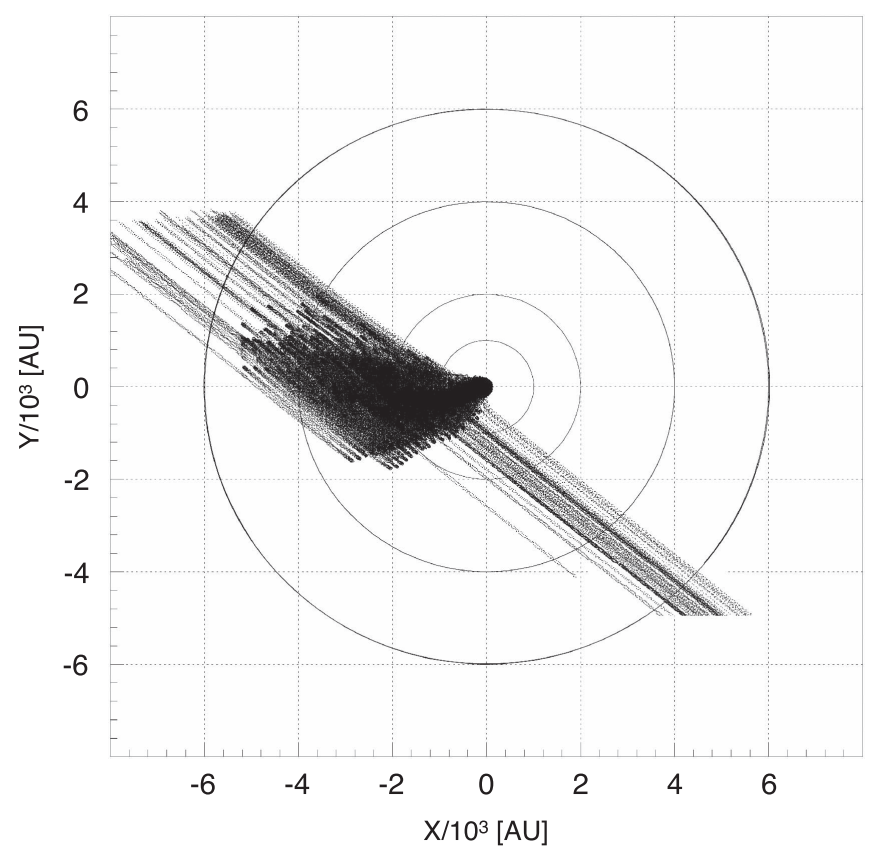

Figure 3. Integrated trajectories of protons with energy of $1 \mathrm{TeV}$, starting from Earth with initial uniform direction distribution, calculated with the heliospheric magnetic field of Figure 1. The figure illustrates the complex structure of more than 100 trajectories passing through the heliosphere and ultimately streaming along the uniform interstellar magnetic field. The regions where the trajectories cross the injection sphere of radius 6000 au are used to identify where to forward-propagate cosmic-ray particles (see text). Note that on the interstellar-wind downstream direction (i.e., in the upper left corner of the figure), particles are more spread out in space as an effect of the elongated heliospheric tail, compared with those in the upstream direction (i.e., in the lower right corner of the figure).

The low-rigidity particle group corresponds to $\left\langle r_{L}\right\rangle \sim 70-100$ au (i.e., smaller than the size of the heliosphere). For a large fraction of particles, $r_{L}$ is the same order of magnitude as the magnetic instabilities on the heliospheric boundary. Note the wide distributions of $r_{L}$ in Figure 4 are due to the changes in magnetic field and pitch angle as particles propagate through the heliosphere. The high-rigidity group corresponds to $\left\langle r_{L}\right\rangle \sim$ 700-1000 au (i.e., just larger than the heliosphere transverse size but smaller than the predicted heliospheric tail length). As discussed in DL13, the rigidity scale of $10 \mathrm{TV}$ is when the heliospheric effect on the particle distribution starts to become subdominant, compared with that from the ISM. This rigidity scale corresponds to $\mathrm{CR}$ particles in the range of $10-300 \mathrm{TeV}$, depending on the mass. 

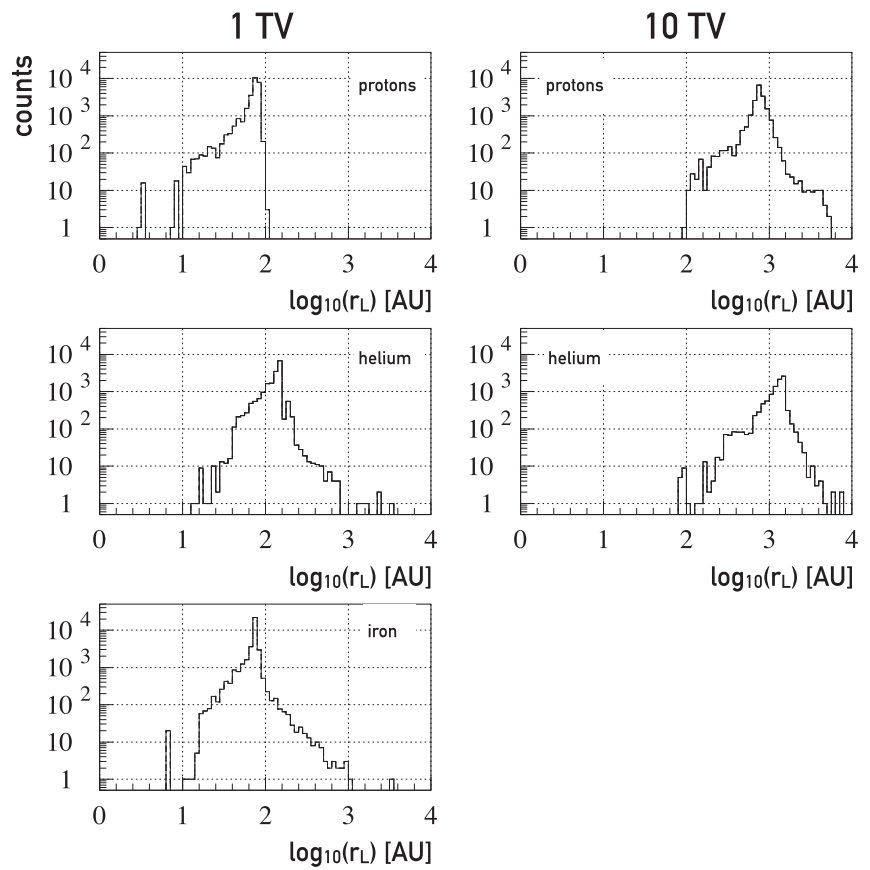

Figure 4. Distributions of instantaneous gyroradii $r_{L}$ (in units of au) of the particles from sets of Table 1, calculated along their trajectories. Note the wide range of variabilities of $r_{L}$ due to the changes in magnetic field and pitch angle as particles propagate through the heliosphere.

\section{The Validity of Liouville's Theorem}

As discussed in Section 3.2, there are two possible ways to obtain the anisotropy at Earth. One involves the application of Liouville's theorem to link the distribution of the particles at some distance in the ISM to the arrival distribution at Earth. The other is to directly propagate the particles from outer space and record the particles' positions at Earth.

In LX16, the theoretical framework for the application of Liouville's theorem in the study of CR-arrival direction was provided. The theorem states that the particle density in the neighborhood of a given system in phase space is constant if restrictions are imposed on the system (Goldstein et al. 2002). We obtained the equation

$$
\frac{\partial \rho}{\partial t}+\boldsymbol{v} \cdot \boldsymbol{\nabla}(\rho)+\boldsymbol{F} \cdot \nabla_{p}(\rho)=\frac{d \rho}{d t}=0,
$$

which is precisely the expression for Liouville's theorem (Goldstein et al. 2002; Bradt 2008), where $\boldsymbol{\nabla}_{p}$ is the del operator in momentum space, $\rho$ is the distribution function, and $\boldsymbol{F}$ is the applied external force.

The most relevant conditions for its application, as shown in LX16, are that the number of particles is conserved and that the forces acting on the particles are p-divergence-free. This last restriction tells us that the forces have to be conservative and differentiable. Collision processes evidently violate this condition. In addition, Liouville's theorem can be considered in the context of conservation of information. Each time that a collision event occurs, it violates the connectedness, and information is lost. Therefore particle trajectories cannot be time reversed.

The derivation provided in LX16 for Equation (3) is for a pure magnetic force, but in fact, when calculating particles' trajectories in a magnetic field subject to perturbations and instabilities, a variety of factors come into play. The most significant effect is when particles encounter a region where the magnetic field varies abruptly (i.e., the scale of variation of the magnetic field is shorter than the gyroradius of the particle). In this scenario, the trajectory does not have time to adjust smoothly to this change, and the scattering process can be effectively considered a collision. For such cases, the righthand side of Equation (3) can be modified by the addition of a term, $\left[\frac{\partial \rho}{\partial t}\right]_{c}$, which takes into account collisions of various origins that are differentiated by their exact functional form, given the fact that they will cause a nonzero time rate of change in the distribution function (Baumjohann \& Treumann 1996). Under these conditions, Liouville's theorem cannot be applied.

To test the abruptness in particle trajectories, it is possible to calculate how the density in phase space is modified by scattering processes (i.e., how adiabatic the change is). In the presence of collisions, the magnetic moment of the gyrating particles changes. Therefore, to check for the adiabaticity of the trajectories, we can calculate the magnetic moment for each particle at each time step and find out if, statistically, it truly behaves as an adiabatic invariant. The relativistic magnetic moment (also called first adiabatic invariant) is given by

$$
\mu=\frac{p_{\perp}{ }^{2}}{2 m|\boldsymbol{B}|},
$$

where $p_{\perp}$ is the momentum perpendicular to the magnetic field $\boldsymbol{B}$ and $m$ is the particle mass. This quantity, relating magnetic field and perpendicular momentum of the particle, is conserved if the field gradients are small within distances comparable to the particle gyroradius. Note that no assumption about the conservation of magnetic moment is used in the numerical integration calculation.

To perform a statistical test on the first adiabatic invariant, we integrated trajectories from two different data sets, each with $1 \times 10^{4}$ particles, initiated at Earth and back-propagated to outer space. One set corresponded to $1 \mathrm{TeV}$, and the other corresponded to $10 \mathrm{TeV}$ protons. Using these specific sets, the magnetic moment in Equation (4) was calculated at each integration time step and plotted in a histogram. The mean value $\bar{\mu}$ of the magnetic moment of the particle ensemble from each data set and the corresponding standard deviation $\sigma_{\mu}$ were calculated. Figure 5 shows the ratio $\sigma_{\mu} / \bar{\mu}$ for the two sets. A distribution with $\sigma_{\mu} / \bar{\mu}=0$ indicates that the conservation of the magnetic moment is perfect. In this case, particles can mirror back and forth between magnetic bottles, which maintain the magnetic moment conserved. However, a distribution peaked at a value much larger than one means that the particles suffer strong variations in their trajectories and collision-like interactions happened. It is not simple mirroring for most particles, but effectively resonant scattering processes at the heliospheric boundary where particles propagate across magnetic field lines (see, e.g., Desiati \& Zweibel 2014) with a stochastically redistributed pitch angle. The distributions obtained for these two sets show peaks at around 5 and 7, with strong skewness to the right, or toward higher values of $\sigma_{\mu} / \bar{\mu}$. This indicates that the magnetic moment fluctuated strongly, that severe changes have occurred, and collision-like interactions happened to the particles under consideration. A stronger deviation in the conservation of magnetic moment is observed with the low-rigidity set compared with the highrigidity set, because of the stronger scattering effects at lower rigidity. Note that in LX16 the same trajectory integration code 


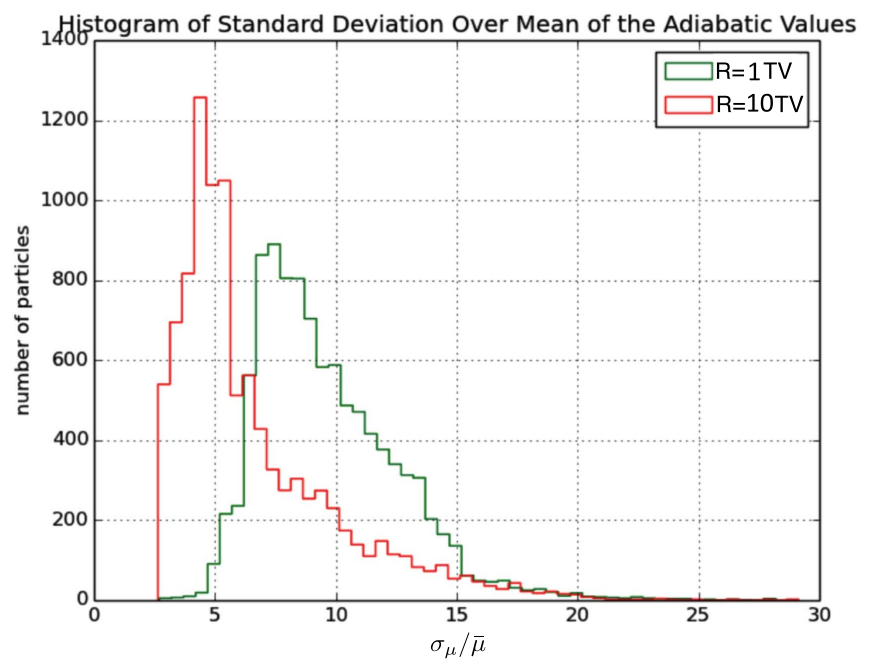

Figure 5. Histogram of standard deviation of magnetic moment $\sigma_{\mu}$ over mean magnetic moment $\bar{\mu}$ for the two rigidity data sets of Table 1 . The red histogram corresponds to the $R=1 \mathrm{TV}$ (p, He, Fe) mixed composition set, and the green histogram to the $R=10 \mathrm{TV}$ (p, He) mixed composition. The magnetic moment is calculated for each particle at all time steps. The mean value and the standard deviation are for the total trajectory.

was used to calculate particle trajectories in compressible MHD turbulence, in the rigidity range between $750 \mathrm{TV}$ and $30 \mathrm{PV}$. In that case, it was found that the ensemble average was $\left\langle\sigma_{\mu} / \bar{\mu}\right\rangle \lesssim 1$ (i.e., significantly smaller than in the present case). The accuracy of the numerical trajectory integration is at the same level as that in LX16. The adaptive time step algorithms constrain both spatial and momentum coordinates to the same relative error level, thus limiting accuracy in spatial coordinates to a level that is $\ll r_{L}$, even after several tens of thousands of gyrations. Therefore the strong nonconservation of the ensemble-average magnetic moment is not caused by lack of numerical accuracy but rather by the characteristics of the magnetic field used in this study. The scattering processes with the heliospheric magnetic instabilities determine the global statistical properties of the particles. The value of $\left\langle\sigma_{\mu} / \bar{\mu}\right\rangle$ in Figure 5 is smaller in the higher rigidity set, because scattering is less effective in redistributing particles with a gyroradius significantly larger than the spatial size of the instabilities.

To conclude, interactions with the heliospheric magnetic field model used in this study result in dramatic changes in the distribution of particle trajectories. The original directional information carried by the particles is lost in these collision-like events. Trajectories diverge due to the magnetic field lines geometry in the regions of magnetic instabilities at the boundary between the heliosphere and the ISM. Thus, based on these considerations, it is not possible to assume that Liouville's theorem is applicable in this case.

For that reason, in this work, the forward-propagation method is used. With this method, anisotropy arises naturally from particle propagation and the interaction with the heliosphere. One important factor to take into account is that since there is a violation of the conditions of Liouville's theorem, we cannot make causal links to or rely in any way on the reversibility of the trajectories; consequently, this constrains the possibility of connecting a specific position in the ISM and the arrival direction at Earth. Therefore it is only possible to determine to what degree the incoming distribution from outer space is distorted due to the features of the heliosphere, but not possible to draw direct correlations between the incoming specific directions and the ones observed at Earth. In our case, we will show how the heliosphere acts on and distorts this distribution, and how anisotropies arise.

\section{Results}

This section shows the results obtained with the numerical calculation data sets described in Section 3.2.

\subsection{Sky Maps of Arrival Direction Distribution}

The numerically integrated trajectories of the sets in Table 1 were combined according to the mixed CR composition from Gaisser et al. (2013; i.e., approximately $40 \%$ protons, $40 \%$ helium nuclei, and $20 \%$ iron nuclei at low rigidity, and $50 \%$ protons and $50 \%$ helium nuclei at high rigidity) and used to study the effects that scattering processes in the heliospheric magnetic field have on the particle arrival direction distributions. As mentioned in Section 4, unlike the procedure followed in LX16, in this study particles were injected in two regions on the injection sphere at 6000 au distance from Earth, aligned along the LIMF and forward-propagated. At each point within those two regions, the particle directions were chosen from a uniform distribution toward the inner sphere. As shown in Table 1, a large fraction of the injected particles does not reach the target sphere, in proximity of Earth, because of scattering processes in the magnetic instability regions, thus contributing to the anisotropic distribution independently of the initial CR density gradient.

The particles hitting the target sphere were recorded and are represented in the sky maps of Figures 6 (for particles injected in the region downstream of the ISM flow, and in proximity of the heliospheric tail) and 7 (for particles injected in the region upstream of the ISM flow). In the figures, the direction of the heliotail is indicated with a yellow star. At the top of the figures, the initial positions on the injection sphere of those trajectories that reach the target sphere are shown. The yellow dashed boxes indicate the region of initial positions of all generated particles. The limited size of those regions show that those particles streaming along the LIMF within a relatively narrow magnetic field-line tube have the highest chance of reaching the target sphere. Note that within those localized regions, all particles have a wide range of uniformly distributed pitch angles (or directions), which determines the size of the corresponding gyroradius $r_{L} \approx(220 / Z)(E / \mathrm{TeV}) \sqrt{1-\mu^{2}}(\mu G / B)$ au, with $\mu$ the cosine of the pitch angle. The instantaneous gyroradius along the particle trajectories are shown Figure 4, and the corresponding mean and rms values are shown in Table 1. At the center of the figures, the arrival directions of the $1 \mathrm{TV}$ rigidity scale particles at the target sphere are shown. The sky maps show that although particles arrive at the heliosphere streaming along the LIMF from one specific direction, they are significantly redistributed. Approximately $50 \%$ of the particles streaming from the downstream direction (and thus approaching the heliosphere in proximity of its elongated tail) undergo multiple scattering processes and appear as if they approach Earth from the upstream region (center of Figure 6), while approximately $20 \%-30 \%$ of the particles streaming from the upstream direction (and thus approaching the heliosphere in the proximity of its nose) appear to approach Earth from the downstream region (center of Figure 7). Resonant scattering along the heliotail has a more pronounced focusing effect toward the inner heliosphere. The wider injection region downstream 

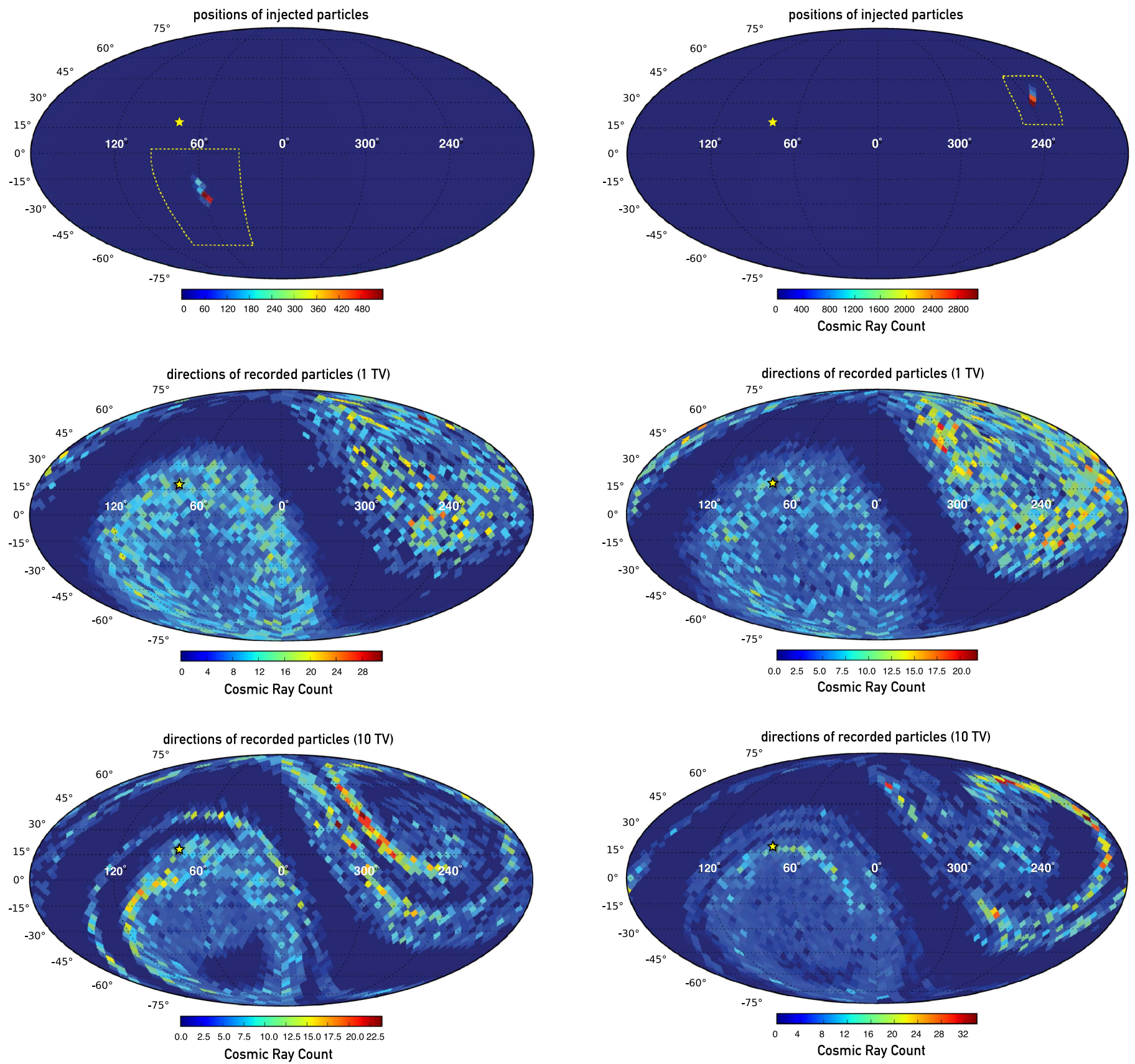

Figure 6. Top: map in equatorial coordinates of the positions of injected particles (from the $60^{\circ} \times 60^{\circ}$ region of the heliosphere upstream of the ISM flow). Only the initial positions of those particles that are actually recorded are shown here. Center: map in equatorial coordinates of the arrival direction distribution of the recorded mixed composition particles at rigidity scale of $1 \mathrm{TV}$. Bottom: map in equatorial coordinates of the arrival direction distribution of the recorded mixed composition particles at rigidity scale of $10 \mathrm{TV}$. The yellow star indicates the approximate position of the heliospheric tail. The dashed yellow box corresponds approximately with the region of initial position of all the particles.

(top of Figure 6) compared with that upstream (top of Figure 7) shows that the heliotail is able to trap particles more efficiently from larger distances downstream and collect them near Earth.

The arrival directions of the $10 \mathrm{TV}$ rigidity scale particles on the target sphere are shown at the bottom of the figures. The effects of scattering on magnetic perturbations are still visible; however, the particle distribution is not as smooth, but it develops localized regions associated with the magnetic field

Figure 7. Top: map in equatorial coordinates of the positions of injected particles (from the $30^{\circ} \times 30^{\circ}$ zone of the heliosphere downstream of interstellar side, in proximity of the heliotail). Only the initial positions of those particles that are actually recorded are shown here. Center: map in equatorial coordinates of the arrival direction distribution of the recorded mixed composition particles at rigidity scale of $1 \mathrm{TV}$. Bottom: map in equatorial coordinates of the arrival direction distribution of the recorded mixed composition particles at rigidity scale of $10 \mathrm{TV}$. The yellow star indicates the approximate position of the heliospheric tail. The dashed yellow box corresponds approximately with the region of initial position of all the particles.

geometric structure. Even at this high rigidity, it is possible to notice that multiple scattering effects of the heliotail are stronger for particles streaming downstream than for those upstream. At higher rigidity, with the decreasing influence of scattering, particle distribution is expected to converge to that at the injection sphere.

A side effect of the relatively large radius of the target sphere (200 au; i.e., 2-3 times the gyroradius at 1 TV rigidity scale) is 

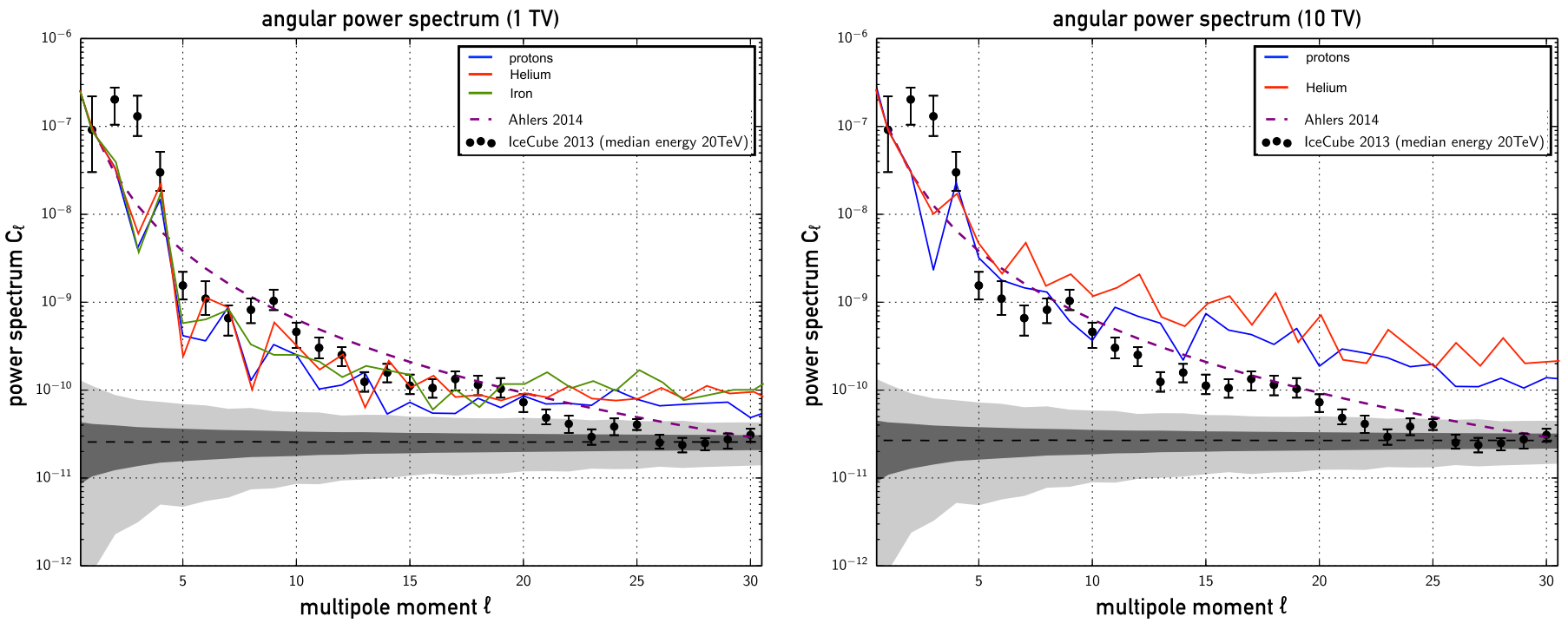

Figure 8. Angular power spectrum of the arrival direction distribution on the target sphere of the $1 \mathrm{TV}$ rigidity particle sets (on the left) and of the $10 \mathrm{TV}$ rigidity particle sets (on the right). Protons (blue lines), helium nuclei (red lines), and iron nuclei (green line) are separately shown. The gray bands show the $1 \sigma$ and $2 \sigma$ bands for a large set of isotropic sky maps. The black circles are the results from the IceCube Observatory at a median energy of 20 TeV (Santander et al. 2013; Aartsen et al. 2016). The dashed purple line is the power spectrum from Ahlers (2014). The angular power spectrum results are normalized to the IceCube experimental results at the dipole component $(\ell=1)$. Note that the angular power spectra are calculated with all particles initiated from both regions on the injection sphere.

that although the trajectories show the effect of multiple scattering processes across the heliospheric boundary (see Figure 3), they are not propagated too deep into the vicinity of Earth. As a consequence, although particles propagate across magnetic field lines with stochastically distributed pitch angle, the connection to the large-scale magnetic field direction is still relatively strong because of the general structure of the heliosphere. This generates very low particle populations along directions that are approximately perpendicular to the LIMF (visible as a dark band at the center and the bottom of Figures 6 and 7).

The figures show a specific snapshot. However, particles reaching Earth are from all masses and energies, and groundbased experiments have relatively poor mass and energy resolutions. Each infinitesimal rigidity interval produces a characteristic fingerprint pattern similar to those in the figures, deeply dependent on the properties of the heliospheric magnetic field. Observations reveal the overlapping of those characteristic distributions, and in fact, there is experimental evidence that coexisting anisotropy features originate from different energy ranges (see, e.g., Bartoli et al. 2013; Aartsen et al. 2016). Even though in this study we provide a window on the possible effects of CR composition, a detailed prediction of the observation requires fine-tuning of several effects, which is not within the scope of this work.

\subsection{Angular Power Spectrum}

As discussed in the previous section, the topology of the sky maps in Figures 6 and 7 is associated with the specific rigidity scales used for the particular particle sets of Table 1. Because of the stochastic nature of scattering processes, statistically uncorrelated data sets would produce similar but not identical sky maps. Even with a seemingly different topology, however, such sky maps would have the same angular power spectrum, since this contains global statistical properties of the ensemble of particles, independent of the spatial location of the anisotropic features. The angular power spectrum, therefore, is a physically relevant quantity to study.

Figure 8 shows the angular power spectrum for the particle sets of Table 1 . On the left are the results for the $1 \mathrm{TV}$ rigidity sets and on the right are the results for the $10 \mathrm{TV}$ rigidity sets. For each particle set, the trajectories propagating from both injection regions are used (see Section 3.4). As expected, the shape of the angular power spectrum depends on particle rigidity. As in LX16, the figures shows the angular power spectrum from the IceCube observatory (Santander et al. 2013; Aartsen et al. 2016), the power spectrum from Ahlers (2014), and that corresponding to an isotropic arrival direction distribution of the same number of particles. In the figures, the power spectra are normalized to each other at the dipole component (i.e., $\ell=1$ ).

$\mathrm{CR}$ particles in the $1 \mathrm{TV}$ rigidity scale, as discussed in Section 5.1, are those most affected by multiple scattering induced by magnetic instabilities at the heliospheric boundaries. Their arrival direction distribution appears to develop angular structures on the order of $20^{\circ}(\ell \approx 10-15$, where it reaches the statistical limit), as shown on the left of Figure 8. In the $10 \mathrm{TV}$ rigidity scale, on the other hand, the small-scale filaments visible in Figures 6 and 7 contribute to the higher power for large values of $\ell$ on the right of Figure 8 . As already mentioned, the sets used in this study represent two particular particle rigidity snapshots of the wide CR energy spectrum. Figure 8 highlights that a complex angular power spectrum arises even when particles propagate across relatively smallscale magnetic structures on a short distance scale. This overlaps with the effects of large-scale turbulence in the ISM over long distance scales (as discussed in LX16). While the ISM contribution is stochastically distributed, the heliospheric effects, although produced by scattering on magnetic instabilities, are expected to have directional correlations with the heliosphere. The experimental separation of the two contributions is the key to exploring the properties of the heliospheric magnetic field with TeV CRs. 


\section{Discussion}

We have shown the dramatic effects that the heliosphere imprints on the CR-arrival distribution at Earth. Our results show that the interactions of CRs with the heliosphere are relevant, and for the 1-10 TV range, cosmic-ray arrival cannot be studied without taking these effects into account.

The feature that distinguishes this work from previous studies is the forward-propagation technique used. We have shown that Liouville's theorem cannot be applied in this case, because resonant scattering on magnetic perturbations on the heliospheric magnetic field generates stochastic pitch angle distributions, and it effectively breaks adiabaticity (Section 4); therefore a back-propagation approach is not applicable. Another important idea behind the forward propagation is the concept of particle escape. As pointed out in Section 3.2 and in Pohl \& Rettig (2015), particle escape due to transport in complex magnetic fields contributes to the resulting arrival direction distribution. Those particles that escape without ever reaching a given target location build up an uneven arrival direction distribution. In a back-propagation approach, there is no particle escape by construction. In this case, a weight function is used to inject an initial anisotropy for the reversed trajectories. The final anisotropy results from the spatial redistribution of a constant particle density.

The results of the present study are different from those in Schwadron et al. (2014), where the importance of a relative large-scale excess of CR (due to a nearby supernova) was highlighted, without accounting for the effects of scattering on the heliospheric magnetic field. In our study, we use a selfconsistent solution to the ideal MHD equations to obtain a model of the heliosphere. In that way, we can assess the direct heliospheric effects on the CR-arrival distribution. And our approach is fundamentally different from the study in Zhang et al. (2014) and Schwadron et al. (2014), where a backpropagation approach was used. In the case of Zhang et al. (2014), a model of the heliosphere was used, although different from the one used in the present study, in that magnetic instabilities were not as prominent. Even though our forwardpropagation approach is computationally expensive, it is the only acceptable technique, since the conditions of Liouville's theorem are not satisfied given the effective collision interactions with the instabilities in the heliosphere (Section 4).

Transport across the galactic magnetic field may be described with homogeneous anisotropic diffusion scenarios, where particles propagate faster along the magnetic field lines. If diffusion describes large-scale CR propagation in the ISM, the arrival direction distribution is expected to have a dipolar shape oriented along the LIMF. Even in the presence of multiple angular scale structures from nondiffusive propagation effects (see Section 1), it is usually assumed that the dipole component still has a direct connection to the underlying largescale diffusion. Observations show that the dipole component appears to be aligned with the LIMF (Ahlers 2016) after accounting for the experimental biasing projection of angular features on the equatorial plane and the limited field of view of all ground-based experiments (Ahlers et al. 2016). The actual direction of the dipole component can be considered accidental and perhaps associated with a relatively recent and nearby source of CRs, contributing to the excess on one side of the LIMF lines rather than the other (see, e.g., Schwadron et al. 2014 and Ahlers 2016).
In reality, the structured interstellar magnetic field, with its different overlapping contributions at different spatial scales, easily generates deviations from the simple diffusion scenario. Particles with a given rigidity are likely to be more strongly affected by magnetic fields with gyro-scale spatial structures. This can cause dramatic changes in the transport properties (see, for instance, Desiati \& Zweibel 2014 and references therein). At a given rigidity scale, CR particle distributions are shaped, over their entire propagation history, by the accumulating effects of magnetic perturbations at scales $\ll r_{L}$, by the magnetic field geometric structure at scales $\gg r_{L}$, and by the strong resonant effects on geometric features at scale $\approx r_{L}$. The LIMF is found to be coherent within approximately 60 parsec (Frisch et al. 2012). This means that CRs approach the heliosphere from the ISM, spiraling around the LIMF lines with pitch angle distribution reflecting their propagation history. At $1 \mathrm{TV}$ rigidity scale, it is thus expected that the heliosphere, with its approximately gyro-scale magnetic instabilities, has the power to redistribute CRs.

The strong heliospheric influence on the arrival direction distribution of CR particles is shown in Figures 6 and 7, where a large fraction of the particles passing through the heliosphere is redistributed in pitch angle by multiple scattering in the magnetic instabilities. While the global anisotropy may still be ordered by the LIMF, the medium- and small-scale features depend on the peculiar properties of the heliospheric magnetic field. In the 1 TV rigidity scale, where resonant scattering processes are more dominant, particles are severely redistributed, and this results in a relatively smoother distribution. On the contrary, in the $10 \mathrm{TV}$ rigidity scale, scattering processes are still significant, but the large-scale average magnetic field induces the formation of medium- and small-scale structures.

In other words, the heliosphere acts as a diffusor where particles with gyroradius $r_{L} \approx L_{\text {instabilities }} \approx 10-100$ au are more stochastically influenced via resonant multiple scattering than those with $r_{L} \gtrsim L_{\text {heliosphere }} \approx 600$ au. A consequence of violation of magnetic moment conservation (see Figure 5) is that an initially uniform pitch angle distribution becomes more structured with most of the energy stored in the large angular scales. The lower the effect of stochastic redistribution from scattering, the higher the contribution from small angular scale structures, as shown in Figure 8. It is likely that the largest angular scale, such as dipole and quadrupole, are affected by these scattering processes as well. However, in this work, such an effect is not explicitly assessed, since particles need to be propagated much closer to Earth.

Statistically uncorrelated data sets can produce sky maps that have similar features; nonetheless, they will not be identical since these scattering processes are stochastic by nature. These sky maps may look different, but they share the same angular power spectrum, since it encloses the global statistical properties of the ensemble, and not the specific spatial locations of the maps' features. Consequently, the physically relevant quantity is the angular power spectrum.

The energy transfer between angular scales is different from that studied in LX16, where the effect of turbulence on a backpropagated particle distribution was considered. In that case, the particle gyroradius $r_{L}$ was always smaller than the largest scale of magnetic turbulence. Therefore, as long as $r_{L}<L_{\text {injection, }}$ particles are always in resonance with a given turbulence scale, with the largest scale conveying more power 
than smaller scales. The energy of an initially dipolar pitch angle distribution is more rapidly transferred into smaller angular scales at higher energy, because resonant scattering occurs on higher power turbulence scales. In both studies, LX16 and this work, it is found that magnetic scattering generates flatter power angular spectra at higher rigidity scales. In general, if scattering is sufficiently strong, it is possible to form small-scale features within a relatively short distance scale as well. In the heliospheric model used in the present study, the size and location of the magnetic instabilities mostly influence $1 \mathrm{TV}$-scale particles, especially those propagating from downstream of the interstellar wind (i.e., in closer proximity to the elongated tail). It is important to note that other heliospheric properties, not accounted for in the model used here, may significantly increase the scattering rate at a specific rigidity range. The 11 year solar magnetic field inversion cycle generates pockets of magnetic polarity dragged outward and along the heliotail by solar wind (Pogorelov et al. 2006). The size of those regions is approximately 200-300 au, as discussed in DL13, which resonate with particles with a rigidity of a few TV. Since the geometry of such polarity regions is different than that of magnetic instabilities, the effect can account for different distributions. This effect was not considered in the present study and will be the subject of a follow-up project.

One carefully studied possibility is that the nonconservation of the magnetic moment discussed in Section 4 may result from poor accuracy of the trajectory interaction code used. The numerical algorithm used to integrate particle trajectories in this study is the same used in LX16, in the study of the MHD turbulence effect of the particle distribution. As extensively discussed in LX16, the Bulirsch-Stoer numerical integration method used is considered one of the best known algorithms satisfying both high accuracy and efficiency (Press et al. 1986) and widely applied in the literature (Giacalone \& Jokipii 1999; $\mathrm{Xu} \&$ Yan 2013). Although this integration method is not symplectic (i.e., it does not have a bound on the global error; see Sanz-Serna \& Calvo 1994), which can then be accumulated at each integration step (Mackay et al. 2006), in this study it was found that the accuracy is still very high (as discussed in the appendix of LX16). The accuracy of the numerical integration is controlled by monitoring the local truncation error estimated at each time step. If the relative error is larger than the relative tolerance level of $10^{-6}$, the step size is adaptively reduced in order to limit the error accumulation in both momentum and spatial coordinates, across the maximum integration time used in this study (between 10,000 and 100,000 gyrations). The accuracy in momentum coordinates was tested, and the resulting energy conservation was found to be constrained well within $10^{-5}$ (see LX16). Due to the adaptive step size, the accuracy in spatial coordinates is $\ll \mathrm{r}_{L}$, thus limiting numerical diffusion to an undetectable level. In LX16, the violation of magnetic moment was not statistically relevant (and therefore the back-propagation method was utilized), while in the current heliospheric study, it is dramatically significant. The difference between the two cases is in the magnetic field used. The numerical accuracy of the trajectory integration method is the same.

The magnetic field interpolation via $3 \mathrm{D}$ cubic spline functions does not appear to reduce numerical accuracy. In general, it is known that a straightforward interpolation of magnetic fields may be not divergence-free, while the calculation of vector potential via Fourier transforms and its interpolation does produce well-behaved magnetic fields. The two different interpolation strategies may influence the particle integrated trajectories, and in particular the first adiabatic invariant (Mackay et al. 2006). However, in the present problem, although the heliospheric magnetic field has instabilities, there are no cusps that may produce significant discontinuities in the interpolated field lines. In addition, the cubic spline interpolation used in this work requires continuity of the second derivative in the entire domain. Therefore it is not expected that a divergence-free interpolation strategy would significantly change the results.

As described in LX16, it is possible to exclude with confidence that the particle trajectory integration method used in this study can induce chaotic behavior as a result of poor accuracy. Therefore it is the opinion of the authors that the effective collisional behavior found in this case is due to the properties of the heliospheric magnetic field used.

To conclude, the study presented in this work explores the effects of the heliosphere on multi-TeV CR-arrival direction distribution. The results illustrate the importance of particle rigidity, $E / Z e$, in making it possible that the heliospheric influence stretches across a relatively wide CR particle energy range. For the particular model of the heliosphere by Pogorelov et al. (2015; described in Section 3.1), resonant scattering processes are strong enough to break adiabaticity and generate stochastic pitch angle distributions. As a consequence, Liouville's theorem could not be applied, and a computationally expensive forward-propagation technique was used. The escape of particles due to magnetic bottle mirroring and multiple resonant scattering generates a rigidity-dependent complex arrival distribution of $\mathrm{CR}$ particles that comprises a wide power spectrum in angular structures. In order to reproduce the observations, proper consideration must be given to a wider range in rigidity, accounting for the actual spectrum and composition of the CRs and especially taking into account that the precise features are not exactly reproducible, given the stochastic process at the heliospheric boundary, and an angular power spectrum approach should be taken in order o study the CR-arrival anisotropy. Assuming that the dipole component of the observed CR anisotropy is the imprint of diffusion in the ISM, the study of the complex angular structure can provide important hints as to the turbulent properties of the ISM (especially at energies $>100 \mathrm{TeV}$, as shown in LX16) and to the properties of the heliosphere (in the TeV energy range).

\section{Summary}

The main results can be summarized as follows:

1. As CRs are strongly affected by magnetic structures on the order of their gyroradius, multi-TeV particles are subject to significant heliospheric scattering. This redistributes CRs and affects their arrival direction distribution. Our work shows that this scattering can have a significant effect.

2. Our simulations show significant resonant scattering of the CRs by the heliosphere. Therefore the conditions of Liouville's theorem are not satisfied and the backwardpropagation technique cannot be used to study $\mathrm{CR}$ anisotropies arising from the interaction of heliospheric magnetic fields with CRs.

3. Our study of the effect of the heliosphere on CR anisotropy, which we performed using the forward-propagation techniques, demonstrates the following features: 
(i) Results for protons: The heliosphere has a strong effect in redistributing CRs at the $1 \mathrm{TV}$ rigidity scale. Multiple scattering with stochastic pitch angle redistribution is relevant, and anisotropy arises even without assuming any weight in the initial arrival distribution of CR particles. The scattering effect is weaker at the $10 \mathrm{TV}$ rigidity scale but is still important in producing small- to medium-angular scale features that contribute to the overall arrival direction distribution.

(ii) Results for heavy nuclei: At $10 \mathrm{TeV}$, the flux of helium nuclei starts to become dominant, while heavy nuclei contribute to about a third of the total flux (Gaisser et al. 2013). We found that high-energy heavy nuclei have an important role in shaping the observed anisotropy at very small angular scales (i.e., multipole moments; $\ell=7-25$ ). This also means that the heliospheric influence affects observed anisotropies over a wide energy range.

4. Our study calls for both more extensive observations of CR anisotropies and more detailed numerical testing using high-resolution models of the heliosphere. Future research should also take into account the significant time variations of the heliospheric magnetic field related to both the 11 year cycle and dynamical instabilities on the longer timescales.

The authors wish to thank colleagues at WIPAC and the Department of Astronomy for discussions on CR anisotropy. We also thank an anonymous referee for useful comments and suggestions. S.X. acknowledges the support from the China Scholarship Council during her stay in University of WisconsinMadison. P.D. acknowledges support from the US National Science Foundation Office of Polar Programs. A.L. acknowledges support of NSF grant AST-1212096, grant NSF-DMS1622457, and NASA grant X5166204101. A.L. and V.L.B. acknowledge support from NASA grant NNX14AJ53G. N.P. was supported by NASA grants NNX14AJ53G, NNX14AF43G, and NNX15AN72G; DOE grant DE-SC0008334; and NSF Petascale Computing Resource Allocation award ACI-1615206.

\section{References}

Aartsen, M., Abbasi, R., Abdou, Y., et al. 2013, ApJ, 765, 55

Aartsen, M., Abraham, K., Ackermann, M., et al. 2016, ApJ, 826, 220 Abbasi, R., Abdou, Y., Abu-Zayyad, T., et al. 2010, ApJL, 718, L194 Abbasi, R., Abdou, Y., Abu-Zayyad, T., et al. 2011, ApJ, 740, 16 Abbasi, R., Abdou, Y., Abu-Zayyad, T., et al. 2012, ApJ, 746, 33 Abdo, A. A., Allen, B., Aune, T., et al. 2008, PhRvL, 101, 221101 Abdo, A. A., Allen, B. T., Aune, T., et al. 2009, ApJ, 698, 2121 Abeysekara, A. U., Alfaro, R., Alvarez, C., et al. 2014, ApJ, 796, 108 Aglietta, M., Alekseenko, V. V., Alessandro, B., et al. 2009, ApJL, 692, L130 Ahlers, M. 2014, PhRvL, 112, 021101

Ahlers, M. 2016, arXiv:1605.06446

Ahlers, M., \& Mertsch, P. 2015, arXiv:1506.05488

Ahlers, M., BenZvi, S. Y., Desiati, P., et al. 2016, ApJ, 823, 10

Amenomori, M., Ayabe, S., Cui, S. W., et al. 2005, ApJL, 626, L29

Amenomori, M., Ayabe, S., Bi, X. J., et al. 2006, Sci, 314, 439

Amenomori, M., Bi, X. J., Chen, D., et al. 2007, Proc. 30th ICRC, (Mérida), 1,593

Amenomori, M., Bi, X. J., Chen, D., et al. 2011, Proc. 32nd ICRC, (Beijing), 1, 137 Avinash, K., Zank, G. P., Dasgupta, B., \& Bhadoria, S. 2014, ApJ, 791, 102 Bartoli, B., Bernardini, P., Bi, X. J., et al. 2013, PhRvD, 88, 082001 Bartoli, B., Bernardini, P., Bi, X. J., et al. 2015, ApJ, 809, 90
Baumjohann, W., \& Treumann, R. A. 1996, Plasma Physics, Magnetohydrodynamics, Kinematics, Radiative Transfer (London: Imperial College Press)

Belov, N. A., \& Ruderman, M. S. 2010, MNRAS, 401, 607

Beresnyak, A., Yan, H., \& Lazarian, A. 2011, ApJ, 728, 60

Blasi, P., \& Amato, E. 2012, JCAP, 1, 11

Borovikov, S. N., \& Pogorelov, N. V. 2014, ApJL, 783, LX16

Borovikov, S. N., Pogorelov, N. V., Zank, G. P., \& Kryukov, I. A. 2008, ApJ, 682, 1404

Bradt, H. 2008, Astrophysics Processes (Cambridge: Cambridge Univ. Press) Brandenburg, A., \& Lazarian, A. 2013, SSRv, 178, 163

Cesarsky, C. J. 1980, ARA\&A, 18, 289

Chalov, S. V. 1996, A\&A, 308, 995

Chandran, B. D. G. 2000, PhRvL, 85, 4656

Cho, J., \& Lazarian, A. 2002, PhRvL, 88, 245001

Cho, J., \& Lazarian, A. 2003, MNRAS, 345, 325

Cui, S. 2011, Proc. 32nd ICRC (Beijing), 1, 6

de Gouveia dal Pino, E. M., \& Lazarian, A. 2005, A\&A, 441, 845

de Jong, J., et al. 2011, arXiv:1201.2621

Desiati, P., \& Lazarian, A. 2012, NPGeo, 19, 351

Desiati, P., \& Lazarian, A. 2013, ApJ, 762, 44

Desiati, P., \& Zweibel, E. G. 2014, ApJ, 791, 51

Drake, J. F., Opher, M., Swisdak, M., \& Chamoun, J. N. 2010, ApJ, 709, 963

Drake, J. F., Swisdak, M., Che, H., \& Shay, M. A. 2006, Natur, 443, 553

Effenberger, F., Fichtner, H., Scherer, K., \& Büsching, I. 2012, A\&A, 547, A 120

Erlykin, A. D., \& Wolfendale, A. W. 2006, APh, 25, 183

Farmer, A. J., \& Goldreich, P. 2004, ApJ, 604, 671

Florinski, V., Jokipii, J. R., Alouani-Bibi, F., \& le Roux, J. A. 2013, ApJL, 776, L37

Florinski, V., Stone, E. C., Cummings, A. C., \& le Roux, J. A. 2015, ApJ, 803,47

Florinski, V., Zank, G. P., \& Pogorelov, N. V. 2005, JGRA, 110, 7104

Frisch, P. C., Andersson, B. G., Berdyugin, A., et al. 2012, ApJ, 760, 106

Gaisser, T. K., Stanev, T., \& Tilav, S. 2013, FrPhy, 8, 748

Galtier, S., Nazarenko, S. V., Newell, A. C., \& Pouquet, A. 2000, JPIPh, 63, 447

Giacalone, J., \& Jokipii, J. R. 1999, ApJ, 520, 204

Giacinti, G., \& Sigl, G. 2012, PhRvL, 109, 071101

Goldreich, P., \& Sridhar, S. 1995, ApJ, 438, 763

Goldstein, H., Poole, C., \& Safko, J. 2002, Classical Mechanics (3rd ed.; San Francisco, CA: Addison-Wesley)

Guillian, G., Hosaka, J., Ishihara, K., et al. 2007, PhRvD, 75, 062003

Hall, D. L., Munakata, K., Yasue, S., et al. 1999, JGR, 104, 6737

Izmodenov, V. V., \& Alexashov, D. B. 2003, AstL, 29, 58

Jansson, R., \& Farrar, G. R. 2012a, ApJ, 757, 14

Jansson, R., \& Farrar, G. R. 2012b, ApJL, 761, L11

Kowal, G., Lazarian, A., Vishniac, E. T., \& Otmianowska-Mazur, K. 2009, ApJ, 700, 63

Kowal, G., Lazarian, A., Vishniac, E. T., \& Otmianowska-Mazur, K. 2012 NPGeo, 19, 297

Kozai, M., Munakata, K., Kato, C., et al. 2014, EP\&S, 66, 151

Kumar, R., \& Eichler, D. 2014, ApJ, 785, 129

Lazarian, A. 2005, in AIP Conf. Proc. 784, Magnetic Fields in the universe: From Laboratory and Stars to Primordial Structures (Melville, NY: AIP), 42 Lazarian, A. 2016, ApJ, 833, 131

Lazarian, A., \& Beresnyak, A. 2006, MNRAS, 373, 1195

Lazarian, A., \& Desiati, P. 2010, ApJ, 722, 188

Lazarian, A., Eyink, G., Vishniac, E., \& Kowal, G. 2015, RSPTA, 373, 40144

Lazarian, A., \& Opher, M. 2009, ApJ, 703, 8

Lazarian, A., \& Vishniac, E. T. 1999, ApJ, 517, 700

Lazarian, A., \& Yan, H. 2014, ApJ, 784, 38

Liewer, P. C., Karmesin, S. R., \& Brackbill, J. U. 1996, JGR, 101, 17119

Lithwick, Y., \& Goldreich, P. 2001, ApJ, 562, 279

Longair, M. 2011, High Energy Astrophysics (3rd ed.; Cambridge: Cambridge Univ. Press)

López-Barquero, V., Farber, R., Xu, S., Desiati, P., \& Lazarian, A. 2016, ApJ, 830,19

Mackay, F., Marchand, R., \& Kabin, K. 2006, JGRA, 111, A06205

Manuel, R., Ferreira, S., \& Potgieter, M. 2014, SoPh, 289, 2207

Mertsch, P., \& Funk, S. 2015, PhRvL, 114, 021101

Munakata, K., Mizoguchi, Y., Kato, C., et al. 2010, ApJ, 712, 1100

Nagashima, K., Fujimoto, K., \& Jacklyn, R. M. 1998, JGR, 1031, 17429

Opher, M., Drake, J. F., Zieger, B., \& Gombosi, T. I. 2015, ApJ, 800, 28

Pogorelov, N. V. 2016, J. Phys. Conf. Ser., 719, 012013

Pogorelov, N. V., Borovikov, S. N., Heerikhuisen, J., \& Zhang, M. 2015, ApJL, 812, L6 
Pogorelov, N. V., Suess, S. T., \& Borovikov, S. N. 2013, ApJ, 772, 2

Pogorelov, N. V., Zank, G. P., \& Ogino, T. 2006, ApJ, 644, 1299

Pogorelov, N. V., Borovikov, S. N., Heerikhuisen, J., \& Zhang, M. 2015, ApJL, 812, L6

Pohl, M., \& Eichler, D. 2013, ApJ, 766, 4

Pohl, M., \& Rettig, R. 2015, Proc. 34th ICRC (The Hague), 451

Potgieter, M. 2013, LRSP, 10, 3

Press, W. H., Flannery, B. P., \& Teukolsky, S. A. 1986, (Cambridge Univ. Press)

Ptuskin, V. 2012, APh, 39, 44

Roberts, P. H. 1956, ApJ, 124, 430

Ruderman, M. S., \& Belov, N. A. 2010, J. Phys. Conf. Ser., 216, 012016

Ruderman, M. S., \& Fahr, H. J. 1995, A\&A, 299, 258

Santander, M., Desiati, P., BenZvi, S., \& Westerhoff, S. 2013, arXiv:1309.7006

Sanz-Serna, J. M., \& Calvo, M. P. 1994, Numerical Hamiltonian Problems (1st ed.; London: Chapman and Hall)

Savchenko, V., Kachelrieß, M., \& Semikoz, D. V. 2015, ApJL, 809, L23

Scherer, K., Strauss, R. D., Ferreira, S. E. S., \& Fichtner, H. 2016, APh, 82, 93

Schwadron, N. A., Adams, F. C., Christian, E. R., et al. 2014, Sci, 343, 988
Shaikh, D., \& Zank, G. P. 2010, PhLA, 374, 4538

Sveshnikova, L. G., Strelnikova, O. N., \& Ptuskin, V. S. 2013, APh, 50 33

Wiedemann, H. 2015, Particle Accelerator Physics (Berlin: Springer)

Xu, S., \& Yan, H. 2013, ApJ, 779, 140

Yan, H., \& Lazarian, A. 2002, PRL, 89, 281102

Yan, H., \& Lazarian, A. 2011, ApJ, 731, 10

Yu, G. 1974, ApJ, 194, 187

Zank, G. P. 1999, in AIP Conf. Ser. 471, Solar Wind 9, ed. S. R. Habbal et al. (Melville, NY: AIP), 783

Zank, G. P., Heerikhuisen, J., Wood, B. E., et al. 2013, ApJ, 763, 20

Zank, G. P., Pauls, H. L., Williams, L. L., \& Hall, D. T. 1996, JGR, 101, 21639

Zank, G. P., Pogorelov, N. V., Heerikhuisen, J., et al. 2009, SSRv, 146,295

Zhang, J. L., Zhang, Y., \& Cui, S. W. 2009, Proc. 31st ICRC, (Łódź), http:// icrc2009.uni.lodz.pl/proc/pdf/icrc0814.pdf

Zhang, M., Zuo, P., \& Pogorelov, N. 2014, ApJ, 790, 5 